\title{
Elective repair of uncomplicated inguinal hernia in south eastern nigerian
}

\author{
Ogbuanya Aloysius Ugwu-Olisa, Emedike Samuel Chukwuemeka Ogadi \\ Department of surgery, Federal Teaching Hospital Abakaliki, Ebonyi State, Nigeria
}

Aims and Objectives: Inguinal hernia repair is the most common operation in general surgery worldwide. Elective repair to safeguard complications is recommended because outcomes of early repair are uniformly superior to results of emergency operations. This study aimed to document our own experiences with regards to elective repair of uncomplicated inguinal hernia and compare our findings with previous data. Materials and Methods: This is a descriptive prospective study of 261 consecutive patients with uncomplicated inguinal hernias repaired electively at Federal Teaching Hospital, Abakaliki, southeastern Nigeria between January 2010 to December 2012. Relevant data extracted and analyzed with SPSS version 22.0 included patients' demographics, clinical details, surgical repairs and outcome measures. Result: A total of two hundred and sixty one patients with 306 uncomplicated inguinal hernias repaired electively were enrolled. The age range of the patients was 2 months 88 years with a mean age of $35.7+/$ SD 16.6 . Majority $(46.4 \%)$ of the patients presented with hernias on the right side, the rest either had hernias on the left(36.4\%) or both sides $(17.2 \%)$. At operation, $203(66.3 \%)$ hernias were indirect, 96 (31.4\%) direct and 7 (2.3\%) pantaloon hernias. Modified Bassini repair was used in the majority (66.7\%) of patients. Complications occurred in only $9.7 \%$ of repairs. At a median follow up of 8 months, $1(0.3 \%)$ hernia recurrence was seen. No mortality was recorded. Conclusion: Inguinal hernias in our environment are very common, but often neglected. Our results show that elective repair is critical for preventing devastating complications and ensuring successful surgical outcomes.

Key words: Hernia, Inguinal, Elective, Repair, Southeast Nigeria
http://nepjol.info/index.php/AJMS DOI: 10.3126/ajms.v7i2.13349 E-ISSN: 2091-0576 P-ISSN: $2467-9100$

\section{INTRODUCTION}

Surgical conditions represent an immense yet underrecognized source of disease burden globally. ${ }^{1}$ The study of such burden constitute a priority research agenda in global surgery. ${ }^{1}$

Across the globe, inguinal hernia is the commonest hernia, accounting for about $75 \%$ of all abdominal wall hernias. ${ }^{1-4}$ Repair of inguinal hernia is the most common operation in general surgery worldwide. ${ }^{2,5-7}$ It is estimated that more than 20 million inguinal hernias are repaired annually around the world; specific rates by country vary from 100 to 300 per
100,000 population per year., ${ }^{8,9}$ Due to poor documentation, the hernia surgery output is largely unknown in Africa, a continent with the greatest burden of the disease compared to the rest of the world. ${ }^{8,10,11}$ Nevertheless, it has been cited that in Africa, an estimated incidence rate of inguinal hernia is $175 / 100,000$ persons each year, but that only 25 are actually repaired. ${ }^{8,10}$

A recent epidemiological study in Ghana shows that at the current repair rate of 30 per 100,000 per year and incidence of symptomatic hernia of 210 per 100,000 per year, a backlog of one million hernias will develop over the next 10 years. ${ }^{1}$ These observations in Ghana probably reflect

Address for Correspondence:

Ogbuanya Aloysius U, General Surgery Team B, Department of Surgery, Federal Teaching Hospital, PMB, 102, Abakaliki, Ebonyi State, Nigeria. E-mail: Ogbuanyaa92@gmail.com. Tel.: +2348033532701

Reprint Request: Dr. Ogbuanya Aloysius U, Department of Surgery, Federal Teaching Hospital, PMB,102, Abakaliki, Ebonyi State, Nigeria. 
the state of the art in most parts of Africa, where much higher percentages of indirect and inguinoscrotal types, emergency cases, morbidities and mortalities following surgery for inguinal hernias have been reported. $2,3,8,12-14$

It has been reported that as high as $20-77 \%$ of inguinal hernia surgery output in Africa present as emergencies compared to 1-3\% seen in Europe and America. ${ }^{2,3,9,12,14-17}$ In Kampala, Uganda, ${ }^{14}$ analysis of 208 groin hernias showed that $195(93.7 \%)$ were inguinal hernias and $81.5 \%$ of these inguinal hernias were of indirect type. Further analysis showed that $54.5 \%$ of the inguinal hernias were strangulated at the time of presentation while significant complications occurred in $41.8 \%$ after repair. ${ }^{14} \mathrm{Mba}^{2}{ }^{2}$ in northwestern Nigeria, examined 227 consecutive patients with inguinal hernia and found that $20.6 \%$ presented as surgical emergencies while $20 \%$ of patients with complicated hernias died with or without surgery.

In our setting, inguinal hernias constitute a major workload for general surgeons, but the need for preventive surgical care is obviously ignored resulting in large accumulated pools of untreated inguinal hernias that have been neglected. Regrettably, it has been cited that numerous deaths occur almost daily in remote rural communities across Africa due to lack of adequate surgical care particularly in patients with long standing untreated hernias complicated with intestinal obstruction and strangulation. ${ }^{8}$ Many previously published studies have recommended prophylactic repair as a safeguard against sequels of late presentations in Africa with added benefits of cost containment and better postoperative outcome measures, ${ }^{2-4,8,10,11}$ yet published data on elective repair of inguinal hernias are scanty in our environment. The aim of this study is to document our own experience with regards to the clinical profile and highlight the surgical treatment and outcome measures of uncomplicated inguinal hernias repaired electively in Abakaliki and compare our results with previous studies.

\section{PATIENTS AND METHODS}

\section{Design and setting}

This is a descriptive prospective study of patients with uncomplicated inguinal hernias repaired electively in Federal Teaching Hospital, Abakalik between January 2010 and December 2012. Federal Teaching Hospital, Abakaliki is located in the southeast geopolitical zone of Nigeria. It is a tertiary, teaching health institution that provides health services for Ebonyi and four other neighboring states in Nigeria.

\section{Subjects}

All consecutive 261 patients who presented with uncomplicated inguinal hernias and subsequently underwent elective hernia repairs were included in this study. Patients who refused to give consent were excluded from the study. Those with strangulated or obstructed hernias were noted, but excluded from further analysis. Recruitment of patients to participate in the study commenced with records of socio-demographic data of each patient in a standard proforma after an informed consent was obtained. The clinical diagnosis of uncomplicated inguinal hernia was made after comprehensive clinical assessment by either a senior registrar or a consultant general surgeon. Also included in the proforma are duration before presentation, type, extent and number of hernia, side affected, primary or recurrence and reducibility of the hernia. Clinical diagnosis, associated problems (hydroceles), types of procedure and anesthesia, early postoperative complications, length of hospital stay and follow up outcomes were recorded.

Basic investigations like hemoglobin level and urinalysis were routinely ordered while focused laboratory tests were reserved for those with co-morbidities. Patients without comorbidities were admitted in the surgical wards on the morning of surgery. Patients with systemic diseases like hypertension, diabetes mellitus and elderly patients were admitted a day before surgery. Preoperative anesthetic review was done, adult patients advised nil per oral regimen and intravenous fluid containing glucose commenced on the morning of surgery. Diabetic and hypertensive patients were managed according to the standard protocols.

All hernia repairs in the pediatric age group were done under general anesthesia. Repairs in the adult patients were performed under general, spinal and local anesthesia depending on patients' physiologic state, extent of disease, surgeons' preference or anesthetist decision. Transverse skin crease incision was routinely adopted. Prophylactic antibiotics were used in the majority of cases. Following exposure and complete separation of the hernia sac, all the pediatric hernias were surgically treated by simple herniotomy. Adult patients received Modified Bassini, nylon darn and mesh repair in that order of frequency. Hydrocelectomy, mostly Lord's procedure was added to repairs in adult hernias with associated hydroceles. The posterior wall repair was routinely performed using nylon-2. Skin closure was executed by the subcuticular or simple interrupted method using nylon $2 / 0$, or vicryl $2 / 0$. Operative findings were noted and postoperative guidelines were clearly written out.

Daycare patients were discharged home on same day if deemed fit to go home safely. Short postoperative clinic visits were arranged for the daycare patients in addition to establishment of functional communication network like telephone contact. Postoperative course and findings were routinely recorded for both daycare and inpatient cases. 
Inpatients were discharged after a variable period of 1-8 postoperative days. Available patients were followed up periodically for two years.

\section{Statistical data analysis}

Data was initially entered on a special feasibility form. Subsequently, statistical data analysis was done using Statistical Package for Social Sciences (SPSS) software version 22.0 (IBM, USA 2015). Categorical variables are reported as percentages. Mean, median and standard deviation were used to summarize continuous variables. Chi-square test was used to determine the $\mathrm{p}$-values for categorical variables, with a $\mathrm{p}$-value of less than 0.05 considered to constitute a statistically significant difference.

\section{Ethical consideration}

Ethical approval to conduct the study was obtained from the Teaching hospital's "Research and Ethical Committee" before commencement of the study.

\section{RESULTS}

During the 3 year period under study, 454 (16.3\%) out of a total of 2,788 general surgical patients seen in the clinics and emergency units of the hospital had external abdominal wall hernias. Of the hernia group, 355 (78.2\%) patients harbored inguinal hernias, representing $12.7 \%$ of the entire general surgery output during the study period. Among the patients with inguinal hernias, 261 (73.5\%) had elective repair while the rest either defaulted from treatment after initial diagnosis $(29,8.2 \%)$, had emergency repair $(62,17.5 \%)$ or died before surgical repair $(3,0.8 \%)$. The patients whose hernias were repaired electively formed our study cohorts.

Further analysis of the 261 patients showed an age range of 2 months to 88 years with a mean of $35.7+/-$ SD 16.6. There were 211 males $(80.8 \%)$ and $50(19.2 \%)$ females with a male to female ratio of $4: 1(\mathrm{p}<0.05)$. The peak age incidence was at 21-30 years age range after which the frequency progressively decreased (Figure 1).

The greater majority $(98,37.5 \%)$ of the patients were farmers, followed by the group comprising of under-five years and primary school pupils, $18.8 \%$ (49 patients). Unskilled laborers accounted for 16.5\% (43 patients). The duration of symptoms prior to presentation ranged from 2 weeks to 29 years with a mean of $6.8+/-$ SD 7.3 . Though nearly half $(46.7 \%, 122)$ of the patients presented within 5 years of noticing the symptoms, only $20.3 \%$ (53 patients) did so within the first one year. Only 3.8\% (10 patients) presented within one month of onset of symptoms while an impressive $50.2 \%$ (131 patients) waited between 6 to 20 years before presentation.
Fifty three(20.3\%) out of a total of 261 patients were in the pediatric age group, $42(79.2 \%)$ males and $11(20.8 \%)$ females while 208 patients were adults, 169 (81.3\%) males and $39(18.7 \%)$ females. Majority $(46.4 \%, 121)$ of the patients presented with hernias on the right side, another $95(36.4 \%)$ individuals presented with left-sided hernias, the remaining $45(17.2 \%)$ patient harbored bilateral hernias. The total number of hernias, laterality, nature, extent and type of hernias are presented in Table 1. Twenty $(80 \%)$ out of 25 patients with associated hydroceles were male children, the remaining $5(20 \%)$ were adult males.

Nearly two third $(170,65.1 \%)$ of the patients had their hernias repaired by trainee surgeons (senior registrars), the remaining $91(34.9 \%)$ patients had their repairs fixed by the consultant surgeons. All the mesh hernioplasties were performed by consultant general surgeons. The associated hydroceles in children were all repaired by herniotomies while the adult cases necessitated hydroceletomies in addition to modified Bassini repairs done. The methods

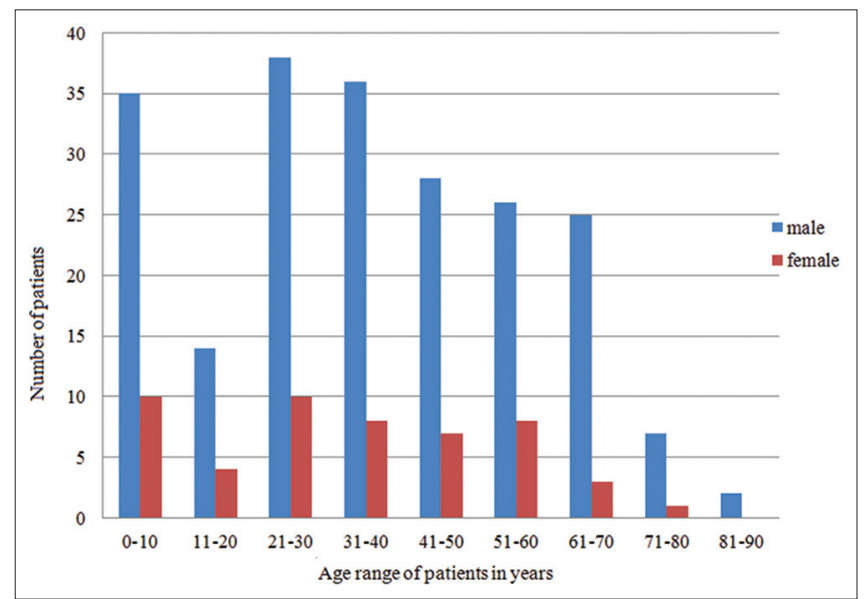

Figure 1: Age and sex distribution of patients with uncomplicated inguinal hernia

\begin{tabular}{|c|c|c|c|c|c|}
\hline \multirow{2}{*}{$\begin{array}{l}\text { Operativel } \\
\text { clinical } \\
\text { findings }\end{array}$} & \multirow{2}{*}{$\frac{\text { Unilateral }}{\text { Right }}$} & Hernia & \multirow{2}{*}{$\begin{array}{c}\text { Bilateral } \\
\text { Right }\end{array}$} & \multirow{2}{*}{$\begin{array}{c}\text { Hernia } \\
\text { Left }\end{array}$} & \multirow{2}{*}{$\begin{array}{c}\text { Total } \\
\text { (percentage) }\end{array}$} \\
\hline & & Left & & & \\
\hline \multicolumn{6}{|l|}{ Nature } \\
\hline Direct & 37 & 28 & 17 & 14 & $96(31.4)$ \\
\hline Indirect & 80 & 65 & 28 & 30 & $203(66.3)$ \\
\hline Pantaloon & 4 & 2 & 0 & 1 & $7(2.3)$ \\
\hline Total & 121 & 95 & 45 & 45 & $306(100.0)$ \\
\hline \multicolumn{6}{|l|}{ Extent } \\
\hline Complete & 51 & 32 & 18 & 13 & $114(37.3)$ \\
\hline Incomplete & 70 & 63 & 27 & 32 & $192(62.7)$ \\
\hline Total & 121 & 95 & 45 & 45 & $306(100.0)$ \\
\hline \multicolumn{6}{|l|}{ Type } \\
\hline Primary & 107 & 89 & 40 & 42 & $278(90.8)$ \\
\hline Recurrence & 14 & 6 & 5 & 3 & $28(9.2)$ \\
\hline Total & 121 & 95 & 45 & 45 & $306(100.0)$ \\
\hline
\end{tabular}


of repair and types of anesthetic techniques employed are shown in Table 2.

The vast majority of the patients $(74.3 \% 194)$ had their anesthesia conducted by anesthetic resident doctors and nurses; the remaining $67(25.7 \%)$ cases were done by consultant anesthetists.

The outcome measures recorded in this study are length of hospital stay, short term and long term complications. The duration of hospital stay ranged between 0 to 8 days with a median of 6 days. Among 10 patients managed on daycare basis, $5(50 \%)$ had repair under local anesthesia, $3(30 \%)$ under spinal anesthesia and another $2(20 \%)$ under general anesthesia. Furthermore, 10 (3.8\%), 33 (12.6\%) and $138(52.9 \%)$ patients were admitted for 1-2,3-4 and 5-6 days respectively. The remaining 70 patients stayed 7-8 days before discharge from hospital. The complications noticed during hospitalization and at the time of surgery were few and minimal, except one case of bladder laceration in adult male with unilateral right inguinoscrotal hernia. This hernia had a sliding component with bladder wall forming part of the hernia sac. The injury was identified intra-operatively and repaired primarily. The postoperative complications recorded after repair of the 306 hernias are shown in Table 3 below.

All the wound infections were superficial; no systemic sepsis was recorded. There was no statistically significant difference between the type of repair technique and wound infection rate $(p>0.05)$, but wound infections were commoner in inguinoscrotal (complete) than inguinal (incomplete) types $(\mathrm{p}<0.05)$. Higher rates of infections

\begin{tabular}{|c|c|c|c|c|}
\hline Procedure & GA & Spinal & LA & Total (\%) \\
\hline Modified bassini & 127 & 36 & 6 & $169(64.8)$ \\
\hline Darning & 25 & 3 & 2 & $30(11.5)$ \\
\hline Herniotomy only & 53 & 0 & 0 & $53(20.3)$ \\
\hline Mesh repair & 1 & 3 & 0 & $4(1.5)$ \\
\hline $\begin{array}{l}\text { Modified bassini and } \\
\text { hydrocelectomy }\end{array}$ & 2 & 3 & 0 & $5(1.9)$ \\
\hline Total $(\%)$ & $208(79.7)$ & $45(17.2)$ & $8(3.1)$ & $261(100.00)$ \\
\hline
\end{tabular}

\begin{tabular}{lcc}
\multicolumn{3}{l}{$\begin{array}{l}\text { Table 3: Postoperative complications after } \\
\text { elective repair of uncomplicated inguinal hernias }\end{array}$} \\
\hline Complication & Frequency & Percentage \\
\hline Wound infection (superficial) & 12 & 3.9 \\
Scrotal hematoma & 6 & 2.0 \\
Seroma & 3 & 1.0 \\
Bladder laceration & 1 & 0.3 \\
Urinary retention & 2 & 0.6 \\
Chronic pain & 5 & 1.6 \\
Recurrence & 1 & 0.3 \\
Total & 30 & 9.7 \\
\hline
\end{tabular}

were also recorded in repairs done by trainee surgeons than wounds of hernias repaired by consultant surgeons. All other short term complications other than the bladder injury were managed conservatively. On long term basis, all five patients with chronic pain improved satisfactorily with analgesics and counseling. Among the follow up patients, only one adult male patient whose bilateral inguinoscrotal hernia was repaired by a trainee surgeon using modified Bassini technique developed recurrence on the right side.

Out of the 261 operated patients, 196 (75.1\%), 156 (59.4\%), $108(41.4 \%)$ and $32(12.3 \%)$ patients were available for follow up at 1 month, 3 months, 6 months and one year respectively. The duration of follow up ranged from 1 to 24 months with a median of 8 months. There was no mortality recorded in this study.

\section{DISCUSSION}

Worldwide, inguinal hernias are very common, particularly in Africa, but the burden of the disease in the continent remains insufficiently described mostly related to negligence engendered by poverty and ignorance. ${ }^{1-3,8} \mathrm{In}$ a recent population based survey in Ghana, the concept of 'Disability Adjusted Life - Years'(DALY) was used to estimate the economic burden needed to treat these hernias. ${ }^{1}$ The researchers estimated that potentially five million DALY would be averted by increasing rate of elective hernia repairs in Ghana alone. ${ }^{1}$ In Africa specifically, it has been proven that elective inguinal hernia repair can be performed cheaply with a DALY costing 12.88 US dollars. ${ }^{10}$ This cost is comparable or more affordable than other disease control strategies already existing in many low and medium income countries (LMICs). ${ }^{18}$ These strategies include insecticide-treated bed nets, oral rehydration salts or antiretroviral therapy, hence the need for consideration of inguinal hernia a functional component of public health system. ${ }^{18}$

The $78.2 \%$ rate of inguinal hernia documented in this study is in common agreement with data in many previously published studies. ${ }^{2-7}$ In Egypt however, a lower frequency of $56 \%$ was reported due to higher relative frequencies of adult umbilical $(22.7 \%)$ and epigastric $(7.4 \%)$ hernias in upper Egypt. ${ }^{19}$ The reasons adduced include high prevalence of chronic liver disease, early marriage and multiple pregnancies common in Egypt. ${ }^{19}$

Generally, inguinal hernia accounts for $10-15 \%$ of operations in general surgery. ${ }^{6,7}$ In our brief general surgical audit, inguinal hernia constitutes $12.7 \%$ of all surgical conditions seen by the general surgeons during the study period making it the most frequently performed operation 
in our unit. Our finding is consistent with the rate of $12.5 \%$ reported in Zaria, ${ }^{20}$ Nigeria and $13.3 \%$ in Tanzania. ${ }^{3}$ A more disturbing figure of $65 \%$ was reported after an audit of the surgical outputs of 8 hospitals in Uganda, Tanzania and Mozambique, prompting the investigators to conclude that there is a great unmet need for surgery in Africa. ${ }^{10}$

In the current report, inguinal hernias showed predilection for male sex and right side, starts early in life and are predominantly indirect and inguinoscrotal. These findings are consistent with reports from previously published data. ${ }^{2-4,6,7,8,20,21}$ Together with a significant bilateral rate of $17.2 \%$, the above pattern supports the congenital basis of some of these hernias that either present early in life commonly as inguinoscrotal or inguinolabial hernias or smolder into late childhood and adult stages before becoming clinically discernible. Elsewhere, it has been cited that the later descent of the right testis into the scrotum in the peri-natal period leaving the processus vaginalis open is the most rational explanation for the right sided preponderance. ${ }^{2,3}$ Four interesting cases of right sided inguinal hernias were observed in the index study; three had previous appendectomies while one individual reported trauma to the right lower abdomen two years previously. These observations highlight the influence of neuromuscular damaging incisions and injuries in the etiology of inguinal hernias previously reported by other workers. ${ }^{2}$ Another peculiarity of inguinal hernia in our setting is the finding that $53.3 \%$ of our study cohorts presented more than 5 years after onset of hernia, similar to a figure of $44.7 \%$ reported in Tanzania. ${ }^{3}$ Our observations that inguinal hernias occur more frequently in farming rural dwellers and other persons with limited resources and health awareness are compounded by distance and poor accessibility to healthcare with subsequent late or emergency presentations in our environment.

In the current study, all the hernia repairs were done by the open method. The modified Bassini repair was used in nearly two-thirds $(66.3 \%)$ of all cases, in agreement with rates of $65.3-79.0 \%$ reported by previous authors. ${ }^{2,3,14,21}$ In the current era of tension free repair in the developed world, modified Bassini method no longer satisfies the requirements of satisfactory hernia surgery, but still remains the standard in Africa. ${ }^{7,10,11,14}$ In a general hospital in Ibadan, Nigeria, Irabor ${ }^{21}$ repaired all the adult inguinal hernias by modified Bassini technique, a further testimony of the popularity of the technique in Africa. Surgeons preference, simplicity, affordability, ease of execution and persistent underfunding of health systems are the reasons for the popularity of Bassini method in Africa. ${ }^{2,3,8,10,11}$ Nevertheless, the time-honored Bassini technique and its various modifications will most likely continue to be relevant in Africa where repairs involving strangulated hernias are common until biologic meshes become available and affordable in Africa. Only 4(1.5\%) patients received mesh repair in this study, higher than the $0.2 \%$ in Tanzania ${ }^{3}$ and $0 \%$ in many other African studies. ${ }^{2,4,14}$ Operation hernia, a European-led hernia aid in Africa, recently reported on a large surgical output of 1748 daycare patients from 8 centers in Nigeria, Ghana and Ivory coast whose inguinal hernias were repaired electively with mesh. ${ }^{10}$ The authors concluded that daycare inguinal hernia repair with mesh is safe in Africa provided the level of sterility during the procedure is high and the surgeon has adequate experience. They further recommended routine use of mesh in elective inguinal hernia repair in Africa. ${ }^{10}$

Surgical site infection with a rate of $3.9 \%$ seen in the index study is the most common complication encountered. This figure is lower than values of $4.5-6.7 \%$ reported previously in similar studies $3,7,14,22$ evaluating complicated and uncomplicated inguinal hernias simultaneously. Interestingly, all the wound infections were superficial and together with other short term outcomes, resolved spontaneously on conservative measures. Only a single major complication involving the urinary bladder was recorded, but recognized intra-operatively and repaired primarily, a condition that probably would have been missed under emergency setting. There is no mortality reported in this study in agreement with previous studies ${ }^{23,24}$ involving uncomplicated inguinal hernias repaired electively. Many studies involving complicated inguinal hernias in Africans have quoted mortality rates of $0.5-40 \% ., 3,7,8$

Despite the limitations imposed by short follow up, the recurrence rate seen in this study is low compared to rates of $1-10 \%$ cited previously. 2,22 A recent study in Turkey ${ }^{25}$ shows that chronic pain occurs in up to $15.4-17.6 \%$ of patients after inguinal hernia repair. The low rate of chronic pain reported in our study could be due to short follow up or under-reporting of symptoms by patients, though similar low rates ranging from $2.9-4.8 \%$ have been reported by other African workers. ${ }^{2,3,22}$

\section{CONCLUSION}

Inguinal hernias are common surgical conditions in our environment. Though the evolution of tension-free repair in our setting is slow, unfavorable features like bilateral, indirect, sliding inguinoscrotal hernias are important predictors of morbidity after elective inguinal hernia repair.

\section{ACKNOWLEDGEMENT}

We are very grateful to the head, department of surgery, Dr Okorie CC and all the consultant general and pediatric 
surgeons in our department for their kind cooperation during the course of this study. We are grateful to Prof Anyanwu SNC for proof reading the manuscript. Special thanks to all the residents of surgery department for their active involvement in data collection. We sincerely appreciate other members of staff of department of surgery for their understanding during this study.

\section{REFERENCES}

1. Beard JH, Oresanya LB, Ohene-Yaboah M, Dicker RA and Harris HW. Characterizing the global burden of surgical disease: A method to estimate inguinal hernia epidemiology in Ghana. World J Surg 2013; 37: 498-503.

2. Mba N. Morbidity and mortality associated with inguinal hernia in Northwestern Nigeria. West Afr J Med 2007; 26(4): 288-292.

3. Mabula JB and Chalya PL. Surgical management of inguinal hernias at Bugando Medical Centre in Northwestern Tanzania: Our experiences in a resource-limited setting. BMC Res Notes 2012; 5: 585

4. Awe JAA, Ugwi $V$ and Omon EH. Inguinal hernias: Analysis of incidence, diagnosis and management of 172 consecutive adult cases at Igbinedion University Teaching Hospital Okada, Nigeria. Glo Adv Res J Med Sci 2014; 3 (7): 168 - 172.

5. Kulacoglu $\mathrm{H}$. Current options in inguinal hernia repair in adult patients. Hippokratia 2011; 15 (3): 223 - 231.

6. Oribabor FO, Amao OA, Akanni OS and Fatidinu SO. The use of nontreated mosquito net mesh cloth for a tension free inguinal hernia repair: Our experience. Nig J Surg 2015; 21 (1): 48 - 51.

7. Adesunkanmi ARK, Badmus TA and Ogundoyin O. Determinants of outcome of inguinal herniorrhaphy in Nigerian patients. Ann Coll Surg H K 2004; 8: 14 - 21.

8. Ohene-Yeboah $M$ and Abatanga $F$. Inguinal hernia disease in Africa: A common, but neglected surgical condition. West Afr $J$ Med 2011; 30 (2): $77-83$.

9. Bay - Nielsen M, Kehlet H, Strand L, Malmastrǿm J, Andersen F, Wara P, et al. Quality assessment of 26,304 herniorrhaphies in Denmark: A prospective nationwide study. Lancet 2001; 258: $1124-1128$.

10. Warwick A, Oppong $C$, Boateng DB and Kingsnorth $A$. Inguinal hernia repair is safe in Africa. East \& cent Afr J Surg 2013; $18(2): 14-17$
11. Kingsnorth AN, Clarke MG and Shillcutt SD. Public health and policy issues of hernia surgery in Africa. World J Surg 2009; 33:1183 - 1193

12. Sanders DL, porter CS, Mitchell $K C D$ and Kingsnorth $A$. A prospective cohort study comparing the African and European hernia. Hernia 2008; 12: $527-529$.

13. Sheshe AA. Feasibility of elective mass hernia repair in Kano State, North western Nigeria. Niger J Basic Clin Sci 2014; 11: $30-35$.

14. Odula PO and Kakande I. Groin Hernia in Mulago hospital, Kampala. East and Cent Afr J Surg 2004; 9: 48 - 52.

15. Ohene - Yeboah $M$ and Dally CK. Strangulated Inguinal hernia in adult males in Kumasi. Ghana Med J 2014; 48(2): 101 - 105.

16. McConkey SJ. Case series of acute abdominal surgery in rural sierra leone. World J Surg 2002; 26 (4): $509-513$.

17. Ohene - Yeboah M, Abatanga F, Oppong J, Togbe B, Nimako B, Amoah $\mathrm{M}$, et al. Some aspects of the epidemiology of external hernias in Kumasi, Ghana. Hernia 2009; 13 (5): 529 - 522.

18. Stewart B, Khanduri P, McCord C, Ohene-Yeboah M, Uranues S, Vega Rivera F, et al. Global disease burden of conditions requiring emergency surgery.BJS 2014;101:9-22.

19. Ammar SA and Ismail T. Abdominal wall hernias in upper Egypt: A different spectrum. East \& central Afr J Surg 2008; 13 (2): $109-114$.

20. Garba ES. The pattern of adult external abdominal wall hernias in Zaria, Nigeria. Nig J Surg Res 2000; 2 (1): 12 - 15.

21. Irabor DO. Hernia repair under local or intravenous ketamine in a tropical low socio-economic population. West Afr J Med 2005; 24: $143-146$

22. Olasehinde OO, Adisa AO, Agbakwuru EA, Etonyeaku AC, Kolawole OA and Mosanya AO. A 5 year review of darning Technique of inguinal hernia repair. Niger J Surg 2015; 21: $52-55$.

23. Usang UE, Sowande OA, Adejuyigbe $\mathrm{O}$, Bakare $\mathrm{TI}$ and Ademuyiwa OA. Day case inguinal hernia surgery in Nigerian children: Prospective study. Afr J Pead Surg 2008; 5: $76-78$

24. Ramyil VM, Iya D, Ogbonna BC and Dakum NK. Safety of day care hernia repair in Jos, Nigeria. East Afr Med J 2000; 77: $326-328$

25. Salih EO, Isin UC, Hatim YU, Suleyman O, Kamil G and Hasan TA. The long term changes in pain-related symptomatology of inguinal hernia following two different herniorrhaphy techniques. Turk J Med Sci 2015; 45: $202-207$.

\section{Authors Contribution:}

OAU - conceptualized the study and performed the literature search, coordinated the manuscript writing, data collection and analysis. He participated in manuscript editing and did the submission; ESO - participated in data analysis, manuscript writing and editing. Both authors read and approved the manuscript for submission.

Authors Information:

OAU is a consultant general surgeon, Department of Surgery, Federal Teaching Hospital Abakaliki. ESO is Chief consultant general surgeon, Federal Teaching Hospital, Abakaliki and Associate professor of surgery, Faculty of Health Sciences, Ebonyi State University, Abakaliki, Nigeria.

Source of Support: Nil, Conflict of Interest: None. 